\title{
Optical modeling for a laser phased-array directed energy system
}

\author{
Gary B. Hughes ${ }^{\mathrm{a}}$, Philip Lubin ${ }^{\mathrm{b}}$, Janelle Griswold ${ }^{\mathrm{b}}$, Brianna Cook $^{\mathrm{b}}$, Durante Bozzini ${ }^{\mathrm{a}}$, \\ Hugh O'Neill ${ }^{\mathrm{c}}$, Peter Meinhold ${ }^{\mathrm{b}}$, Jonathan Suen ${ }^{\mathrm{b}}$, Johanna Bible ${ }^{\mathrm{b}}$, Jordan Riley ${ }^{\mathrm{b}}$, \\ Isabella E. Johansson ${ }^{\mathrm{d}}$, Mark Pryor ${ }^{\mathrm{e}}$ and Miikka Kangas ${ }^{\mathrm{b}}$ \\ gbhughes@,calpoly.edu \\ ${ }^{a}$ Statistics Department, California Polytechnic State University, San Luis Obispo, CA 93407 \\ ${ }^{\mathrm{b}}$ Physics Department, University of California, Santa Barbara, CA 93106 \\ ${ }^{c}$ Physics Department, California Polytechnic State University, San Luis Obispo, CA 93407 \\ ${ }^{\mathrm{d} C}$ Columbia University, New York, NY 10027 \\ ${ }^{\mathrm{e}}$ Vorticy Inc., San Diego, CA 92121
}

\begin{abstract}
We present results of optical simulations for a laser phased array directed energy system. The laser array consists of individual optical elements in a square or hexagonal array. In a multi-element array, the far-field beam pattern depends on both mechanical pointing stability and on phase relationships between individual elements. The simulation incorporates realistic pointing and phase errors. Pointing error components include systematic offsets to simulate manufacturing and assembly variations. Pointing also includes time-varying errors that simulate structural vibrations, informed from random vibration analysis of the mechanical design. Phase errors include systematic offsets, and timevarying errors due to both mechanical vibration and temperature variation in the fibers. The optical simulation is used to determine beam pattern and pointing jitter over a range of composite error inputs. Results are also presented for a $1 \mathrm{~m}$ aperture array with $10 \mathrm{~kW}$ total power, designed as a stand-off system on a dedicated asteroid diversion/capture mission that seeks to evaporate the surface of the target at a distance of beyond $10 \mathrm{~km}$. Phase stability across the array of $\lambda / 10$ is shown to provide beam control that is sufficient to vaporize the surface of a target at $10 \mathrm{~km}$. The model is also a useful tool for characterizing performance for phase controller design in relation to beam formation and pointing.
\end{abstract}

Keywords: DE-STAR, Directed Energy, Laser Phased Array, Planetary Defense

\section{INTRODUCTION}

\subsection{DE-STAR System Concept}

Recent studies have considered the use of directed energy for planetary defense against asteroid impact. DE-STAR, Directed Energy System for Targeting of Asteroids and exploRation, is a phased array consisting of a planar array of laser fiber amplifiers and associated optical elements, and powered by photovoltaics. ${ }^{1,2}$ The main objective of DESTAR is to use focused directed energy to deliver sufficient photon flux to vaporize a spot on the surface of an asteroid. Ejected material produces a reaction force altering the asteroid's orbit, ultimately deflecting the asteroid from its threatening approach. The DE-STAR system depends on phaselocking an entire array of laser emitters. Small arrays would be useful for missions that deploy to an area near the asteroid. ${ }^{3}$ Larger arrays would be capable of operating from considerable distances, allowing consideration of an Earth-orbiting system. An operational DE-STAR would be capable of serving diverse scientific objectives, including spacecraft propulsion ${ }^{4}$, active illumination of asteroids ${ }^{5}$, stand-off composition analysis ${ }^{6}$ and more; these functions are depicted in Fig. 1.

The design cornerstone of DE-STAR is an array of phase-locked laser fiber amplifiers. That is, in order to form and steer a concentrated directed-energy beam, precise phase control of the entire array of lasers

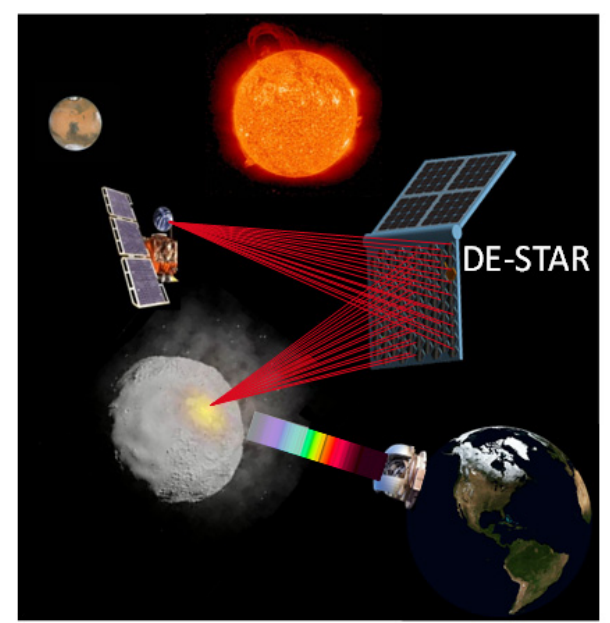

Figure 1. Conceptual diagram of DE-STAR, a proposed laser phased array system for planetary defense and other scientific purposes.

must be possible. Such phase control requires attention to several key aspects of system design that can affect the relative phases of every emitter, including: (1) mechanical alignment of laser and optical components; (2) structural 
vibration; (3) reference phase generation and acquisition; (4) electronic and/or mechanical emitter phase control. Design details for a specific planetary defense mission have been described. ${ }^{7}$ This paper specifically addresses requirements for phase control that are necessary for beam formation and steering in the context of a directed-energy system for stand-off planetary defense.

\subsection{Laser Phased Array Concept}

A baseline design ${ }^{6,7}$ for beam formation and steering is shown in Fig. 2. A seed laser supplies a reference source for the array of fiber amplifiers. Rough pointing of the array to the target is determined by spacecraft attitude control. Fiber tips behind each optical element are mounted to micro-positioner actuators; lateral movement of the laser tips behind each lens provides intermediate pointing adjustment for individual array elements and beam steering. Precision beam steering and beam formation (spot focus) is accomplished by coordinated phase modulation across the array. Feedback from wavefront sensors in front of the optical elements is used to adjust the input phase to each amplifier. This scheme requires a phase reference signal to be present at the exit aperture of each optical element. Ideally, the reference signal would consist of parallel wavefronts travelling along the target axis. Geometric constraints require novel approaches to generation and use of the phase reference signal. ${ }^{7,8}$

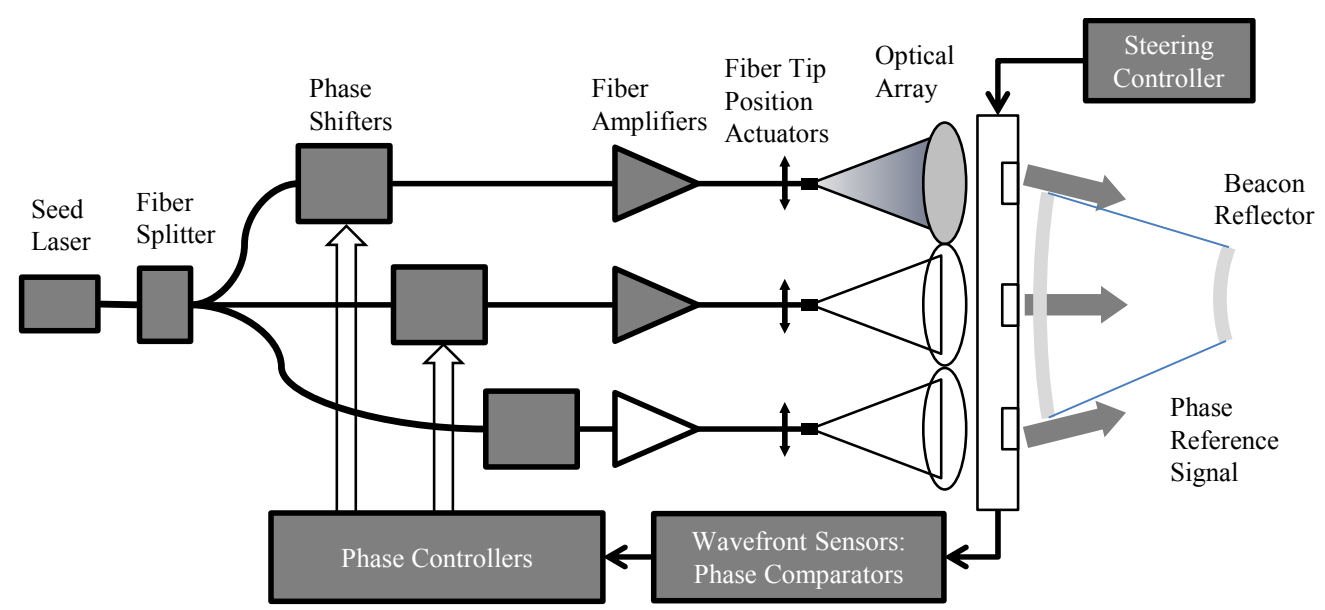

Figure 2. Conceptual block diagram of laser phased array architecture based on kW-class laser fiber amplifiers.

\section{BASELINE LASER PHASED ARRAY SIMULATION}

\subsection{Sum of Complex Frequencies}

The interference pattern and resulting far-field intensity distribution of multiple emitters in a phased-array design can be determined by scalar diffraction theory. Table 1 defines terms commonly used in the antenna literature. ${ }^{8-13}$ For simplicity, simulations in this paper are based on a planar, rectangular array of square emitters with constant, closepacked spacing. Simulations are possible with other geometries by modifying the array pattern contribution.

Table 1. Terms that are commonly used in the antenna literature, including units associated with each term used for simulations presented in this paper.

\begin{tabular}{|c|c|c|}
\hline Symbol & Interpretation & Units \\
\hline$\lambda$ & Nominal emitter wavelength & $\mu \mathrm{m}$ \\
\hline$d$ & Nominal element spacing (array pitch) & $\mu \mathrm{m}$ \\
\hline$\theta$ & $\begin{array}{c}\text { Angular variable (viewing angle away from normal to } \\
\text { emitter array plane) }\end{array}$ & $\mathrm{rad}$ \\
\hline $\mathrm{E}$ & Complex far-field amplitude & $\mathrm{V} / \mathrm{m}$ \\
\hline$I$ & Far-field beam intensity & $\mathrm{W} / \mathrm{m}^{2}$ \\
\hline$k$ & $2 \pi / \lambda$ & $\mu \mathrm{m}^{-1}$ \\
\hline$a$ & Aperture opening size & $\mu \mathrm{m}$ \\
\hline $\mathrm{N}$ & Number of emitters in a single dimension of an array & dimensionless \\
\hline
\end{tabular}


The complex far-field amplitude for a flat, linear array of emitters in phase alignment is given by

$$
E(\theta)=E_{0} \cdot \frac{e^{[i \cdot k \cdot a \cdot \sin (\theta)]}-1}{i \cdot k \cdot \sin (\theta)} \cdot \sum_{p=0}^{N-1} e^{[i \cdot k \cdot p \cdot d \cdot \sin (\theta)]}
$$

Given the complex amplitude, the far-field beam intensity for the linear array is then

$$
I(\theta)=|E(\theta)|^{2}
$$

For a 1-D linear array, the far-field beam intensity for a square array with beam intensity $I_{x}(\theta)$ along one axis and $I_{y}(\psi)$ along a perpendicular axis is

$$
I(\theta, \psi)=I_{x}(\theta) \cdot I_{y}(\psi)
$$

Eqs. 1-3 can be used to determine the far-field intensity pattern of a planar array of emitters. The equation assumes perfect mechanical alignment, as well as perfect frequency, amplitude and phase control for every emitter in the array. A simulation was performed, based on Eqs. 1-3 for a 5 by 5 array. Fig. 3 shows 1-D and 2-D far-field beam patterns.
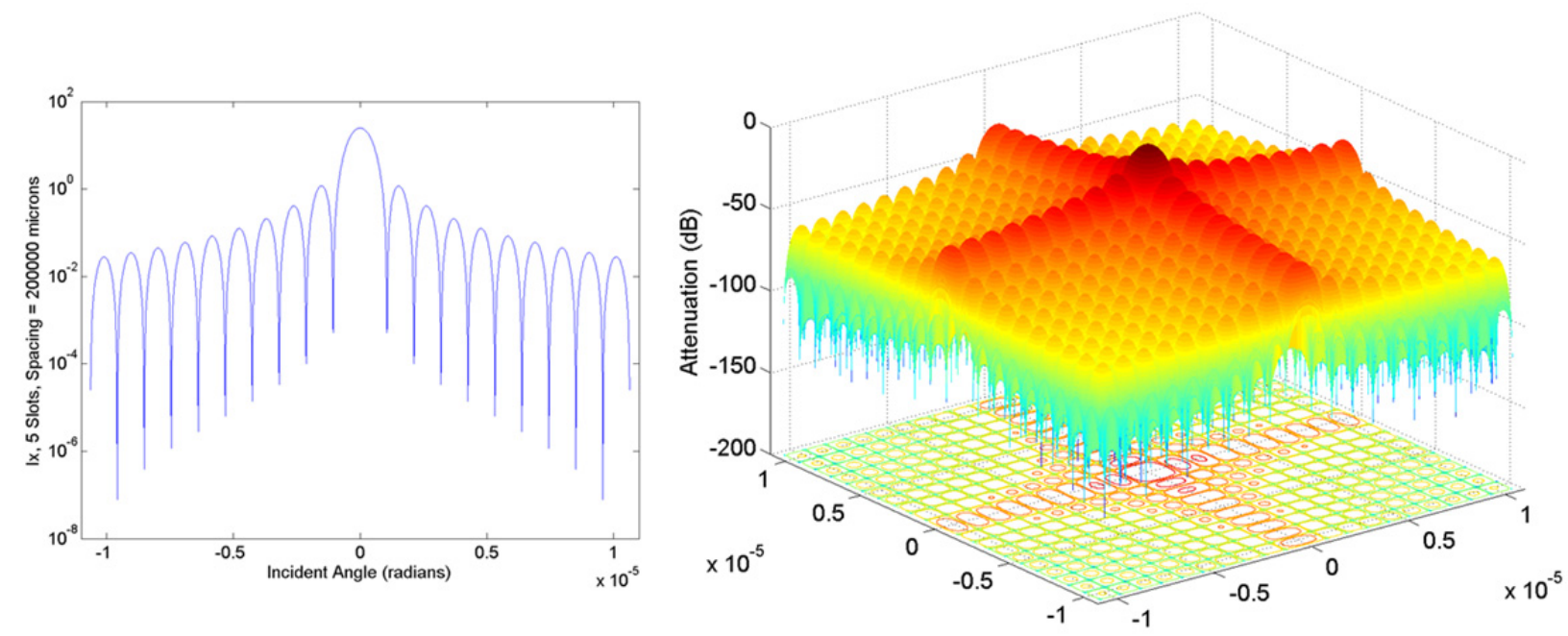

Figure 3. Baseline simulation results for a 5 by 5 close-packed array of square emitters in phase alignment. Each emitters is a $20 \mathrm{~cm}$ by $20 \mathrm{~cm}$ square $(a=d=20 \mathrm{~cm})$, so total aperture is $1 \mathrm{~m}, \mathrm{~N}=25$, and the nominal emitter frequency is set to $\lambda=1.06 \mu \mathrm{m}$. These results would only be attained by having perfect mechanical alignment and perfect frequency, amplitude and phase control of the every emitter in the array.

\section{PHASE PERTURBATIONS}

\subsection{Inclusion of Phase Perturbations in the Optical Model}

Design requirements for a laser phased array can be investigated by introducing perturbations to the baseline optical model given in Eq. 1. In practice, the array will have imperfections in mechanical alignment that will introduce single-emitter pointing errors and phase misalignments. Alignment flaws manifest as static perturbations to the far-field intensity. An operational system will also have vibrational modes, thermal variations and other sources of phase error that contribute temporal perturbations to beam formation and pointing. Fig. 4 depicts the origin of each component on phase alignment. Assembly defects are static, un-correlated phase and amplitude perturbations. Vibrational modes introduce time-varying phase and amplitude perturbations that are correlated across the array. Thermal and other variations contribute to uncorrelated, time-varying phase and amplitude perturbations.

Eq. 1 can be modified to include additive fixed and time-varying phase misalignments, representing the two mechanical scenarios in Fig. 4. The complex far-field amplitude for a linear array of emitters with static $\left(E_{f}\right)$ and timevarying $\left(E_{t}\right)$ phase misalignments at each emitter is given in Eq. 4:

$$
E(\theta, t)=\frac{e^{[i \cdot k \cdot a \cdot \sin (\theta)]}-1}{i \cdot k \cdot \sin (\theta)} \cdot \sum_{p=0}^{N-1} e^{\left\{i \cdot\left[k \cdot p \cdot d \cdot \sin (\theta)+E_{f}(p)+E_{t}(p, t)\right]\right\}}
$$




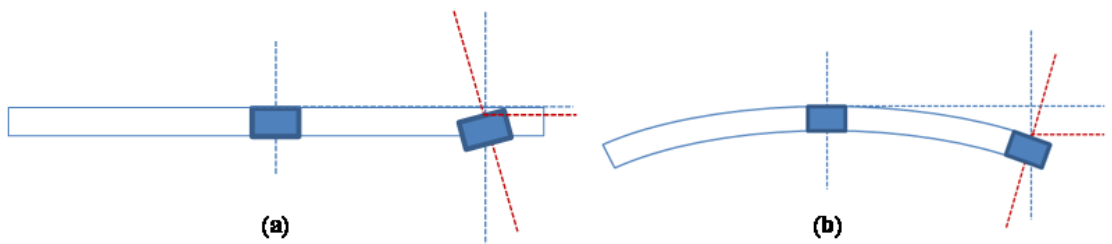

Figure 4. Illustration of the effects on phase alignment of (a) mechanical misalignment of optical elements, and (b) first-mode structural vibration.

\subsection{Generation of Phase Perturbation Terms}

Phase errors due to assembly imperfections are included in Eq. 4 as fixed differences at each emitter, $E_{f}(p)$. Assembly errors can be modeled by random assignment of a fixed phase difference to each element in the array. For the simulations presented in this paper, fixed phase differences are drawn randomly from a normal distribution with zero mean and a specified standard deviation, written as

$$
E_{f}(p)=\operatorname{invNorm}(\operatorname{rand}() \text {, mean }=0 \text {, standard deviation }=\sigma) .
$$

Standard deviation represents the variation among fixed alignment differences, and is typically stated in terms of a fraction of a single cycle at the nominal emitter frequency. Fixed differences of precisely one cycle are phase aligned, so the alignment requirement can be stated as modulo- $1 \lambda$.

Fixed alignment differences can be chosen randomly, since they can be viewed as un-correlated random errors. However, time-varying terms can be both correlated and uncorrelated. Vibrational modes in the structure will create phase misalignments that are correlated among the array emitters. Additional perturbations are also possible, for example due to thermal changes in optical paths or non-linear reactions of optical elements to structural vibrations. Some of these scenarios may result in uncorrelated phase misalignments, which can also be included in the model by adding a series of time-varying terms, i.e., $E_{t}(p, t)$ in Eq. 2 can be a sum representing several components.

\subsection{Simulation Results}

It is well-known that mechanical anomalies on the order of $\sim \lambda / 10$ can introduce significant aberrations in optical systems. The simulation results shown in Fig. 5 represent a laser phased array that includes fixed phase misalignments $\left(E_{f}\right)$ at each emitter with a $1 \sigma$ error of $\lambda / 8$. Comparison of these results to Fig. 3 shows significant beam degradation, with significant power moving from the main peak to side lobes. Also evident is a pointing shift, i.e., the main lobe axis is no longer aligned with the array axis, with a pointing error on the order of $1 \mu \mathrm{rad}$. A time series of pointing errors is shown in Fig. 6.
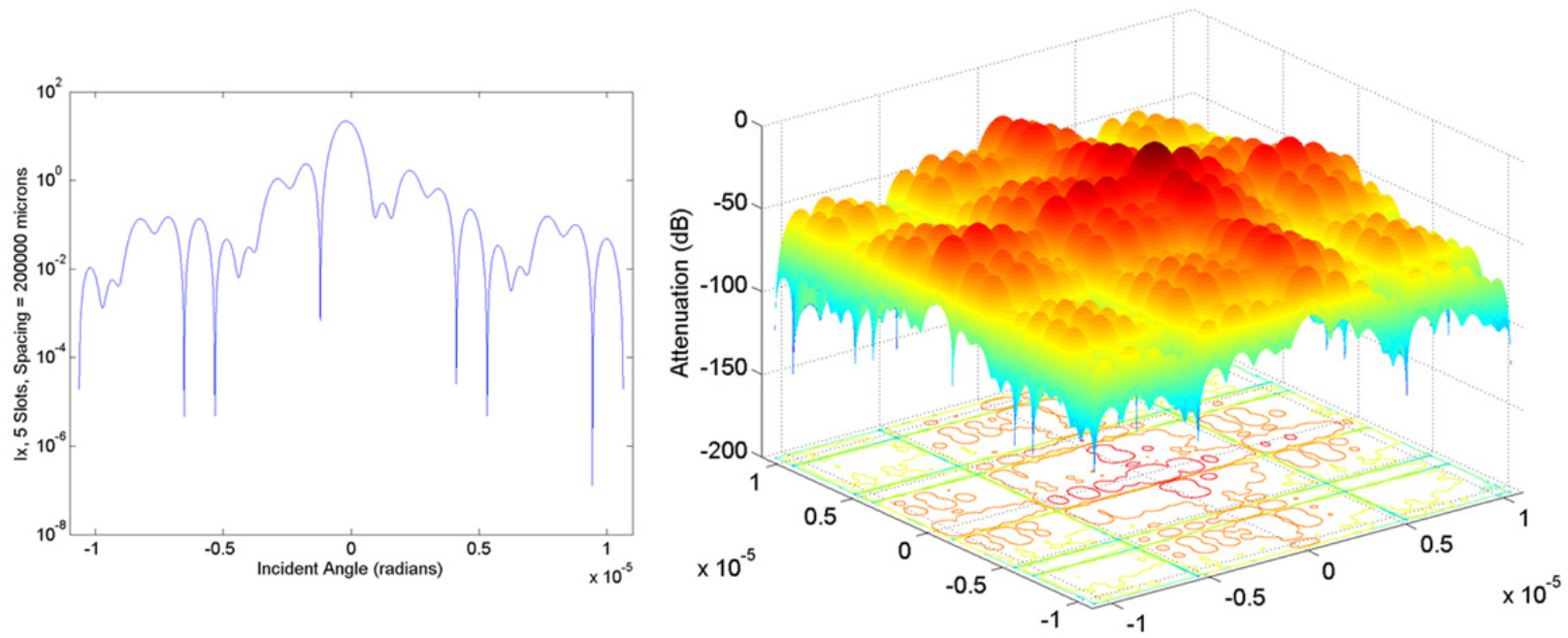

Figure 5. Simulation results for a 5 by 5 close-packed array of emitters with static phase perturbations. Again, emitters are modeled as $a=d=20 \mathrm{~cm}$, total aperture is $1 \mathrm{~m}$, and the nominal emitter frequency is set to $1.06 \mu \mathrm{m}$ (compare to Fig. 3). Mis-alignments were randomly assigned to each emitter, with magnitudes drawn from a normal distribution with $1 \sigma=2 \pi / 8$. 
In addition to static phase misalignments, the model can incorporate time-varying phase errors, such as induced by structural vibration or thermal variations. Fig. 6 shows a simulated time series of pointing error, sampled at $1 \mathrm{kHz}$. As can be seen, the direction of the main lobe is deflected less than $1.8 \mu \mathrm{rad}$. Only uncorrelated phase errors were introduced for the simulation depicted in Fig. 6. Errors were specified as sinusoids with randomly assigned amplitude, frequency and phase, with magnitudes for each sinusoid parameter drawn from uniform distributions on [0, 1]. In the presence of time-varying phase errors, the pointing error is rarely close to $0 \mathrm{rad}$, but always small compared to the main lobe beam width.
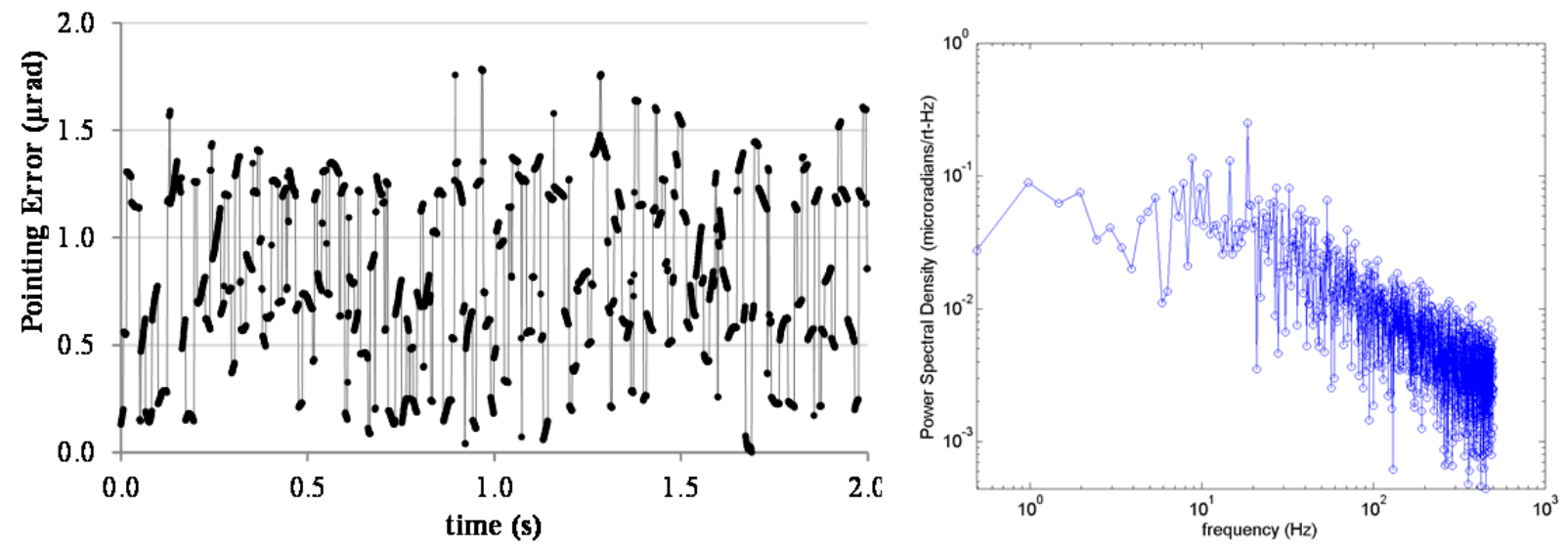

Figure 6. A simulated 2 second time series for a 5 by 5 close-packed array of emitters with un-correlated dynamic phase perturbations across the array. Again, emitters are modeled as $a=d=20 \mathrm{~cm}$, total aperture is 1 $\mathrm{m}$, and the nominal emitter frequency is set to $1.06 \mu \mathrm{m}$. Spectrum calculated from the 2 second time series.

Simulation results can be compared to simple Ruze theory, with one caveat: the comparison is not strictly appropriate, due to assumptions of correlation sizes in Ruze theory. Comparison to Ruze theory provides some amount of model validation. The simulations agree extremely well with the simple Ruze exponential roll off of forward gain or flux on target. The Ruze equation is given in Eq. 5:

$$
\frac{\langle P\rangle}{P_{0}}=e^{-\operatorname{Var}(\varphi)}
$$

where $\operatorname{Var}(\varphi)$ is the variance of the phase per element $\langle\mathrm{P}\rangle$ is the expected value of flux on target with phase perturbations, and $\mathrm{P}_{0}$ is the flux on target with no phase perturbations. Fig. 7 shows results of two Monte Carlo simulations comparing power roll off to Ruze predictions for two arrays.

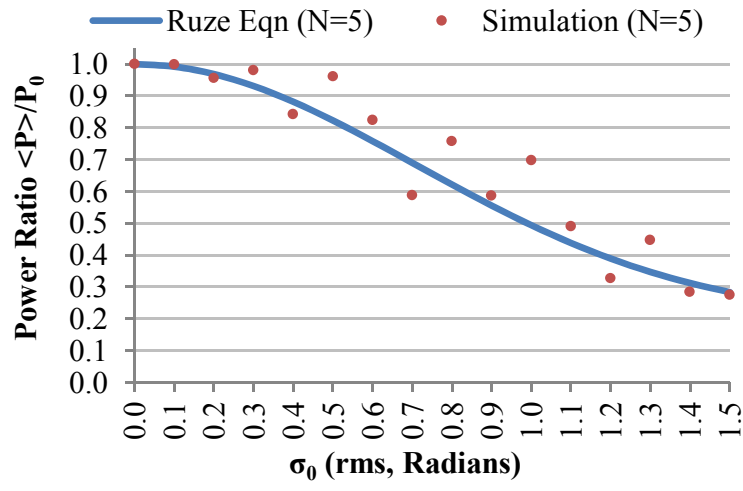

(a)

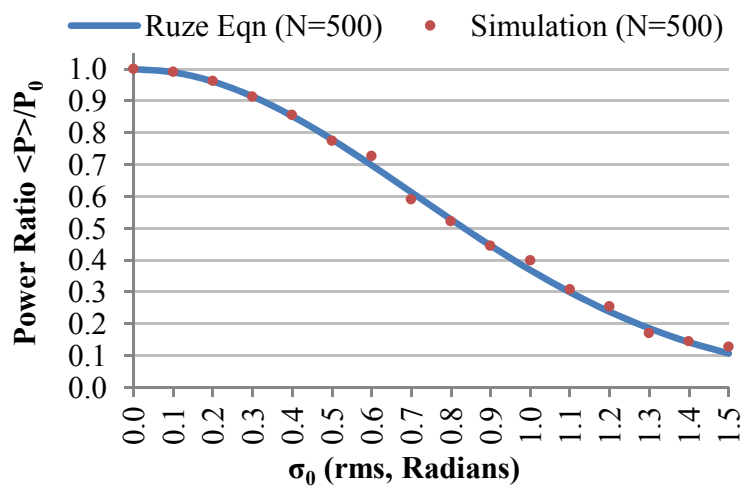

(b)

Figure 7. Comparison of simulation results to the Ruze equation for average gain fall-off as a function of phase error magnitudes across the array. (a) $\mathrm{N}=5$, with $a=b=20 \mathrm{~cm}$ and 10,000 simulation runs; (b) $\mathrm{N}=500$ with with $a=b=1 \mathrm{~m}$ and 10,000 simulation runs. Both plots were created with the nominal emitter frequency is set to $1.06 \mu \mathrm{m}$. 


\section{CONCLUSIONS}

A simulation tool has been developed to explore variations in beam pointing for multi-element laser phased arrays. The simulation is based on a model that incorporates static phase errors, such as could arise from mechanical alignment of array elements. The model also incorporates both correlated and un-correlated time-varying phase errors, such as could arise from structural vibration or thermal variations. The model compares favorably with Ruze theory, providing some measure of model validation.

Results of several scenarios are presented, in particular for a 5 by 5 close-packed array of square emitters with spacing $a=d=20 \mathrm{~cm}$, total aperture of $1 \mathrm{~m}$, and the nominal emitter frequency set to $1.06 \mu \mathrm{m}$. This configuration is intended as a stand-off system for a dedicated asteroid diversion/capture mission that seeks to evaporate the surface of the target at a distance of beyond $10 \mathrm{~km} .{ }^{3}$ For such an array, uncorrelated phase perturbations of $\lambda / 8$ radians resulted in a maximum beam pointing error of less than $1.8 \mu \mathrm{rad}$. At a distance of $10 \mathrm{~km}$, pointing error due to phase perturbations does not move the beam focus outside the nominal full-width, half-max (FWHM) area, maintaining sufficient flux within the target spot to vaporize rocky materials.

These results confirm that phase alignment must be maintained on the order of $\sim \lambda / 10$ to control the main lobe power to within a reasonable fraction of the beam width. Phase control can be specified as modulo- $1 \lambda$. System design must be capable of correcting for both static and dynamic phase perturbations. For dynamic phase errors, feedback control must consider structural modes, and have sufficient bandwidth to correct phased errors induced by smallamplitude vibrations.

\section{ACKNOWLEDGEMENTS}

We gratefully acknowledge funding from the NASA California Space Grant NASA NNX10AT93H in support of this research.

\section{REFERENCES}

[1] Lubin, P., Hughes, G.B., Bible, J., Bublitz, J., Arriola, J., Motta, C., Suen, J., Johansson, I., Riley, J., Sarvian, N., Clayton-Warwick, D., Wu, J., Milich, A., Oleson, M., Pryor, M., Krogen, P., Kangas, M., and O’Neill, H. "Toward Directed Energy Planetary Defense," Optical Engineering, Vol. 53, No. 2, pp 025103-1 to 025103-18 (Feb 2014), doi: 10.1117/1.OE.53.2.025103.

[2] Hughes, G.B., Lubin, P., Bible, J., Bublitz, J., Arriola, J., Motta, C., Suen, J., Johansson, I.E., Riley, J., Sarvian, N., Wu, J., Milich, A., Oleson, M., and Pryor, M. "DE-STAR: phased-array laser technology for planetary defense and other scientific purposes (Keynote Paper)," Nanophotonics and Macrophotonics for Space Environments VII, edited by Edward W. Taylor, David A. Cardimona, Proc. of SPIE Vol. 8876, 88760J (Aug, 2013).

[3] Kosmo, K., Pryor, M., Lubin, P., Hughes, G.B., O’Neill, H., Meinhold, P., Suen, J., C., Riley, J., Griswold, J., Cook, B.V., Johansson, I.E., Zhang, Q., Walsh, K., Melis, C., Kangas, M., Bible, J., Motta, Brashears, T., Mathew, S. and Bollag, J. "DE-STARLITE - a practical planetary defense mission," Nanophotonics and Macrophotonics for Space Environments VIII, edited by Edward W. Taylor, David A. Cardimona, Proc. of SPIE Vol. 9226 (Aug, 2014).

[4] Bible, J., Bublitz, J., Johansson, I.E., Hughes, G.B., and Lubin, P. "Relativistic Propulsion Using Directed Energy," Nanophotonics and Macrophotonics for Space Environments VII, edited by Edward W. Taylor, David A. Cardimona, Proc. of SPIE Vol. 8876, 887605 (2013).

[5] Riley, J., Lubin, P., Hughes, G.B., O’Neill, H., Meinhold, P., Suen, J., Bible, J., Johansson, I.E., Griswold, J. and Cook, B. "Directed energy active illumination for near-Earth object detection," Nanophotonics and Macrophotonics for Space Environments VIII, edited by Edward W. Taylor, David A. Cardimona, Proc. of SPIE Vol. 9226 (Aug, 2014).

[6] Hughes, G.B., Lubin, P., O’Neill, H., Meinhold, P., Suen, J., Riley, J., Johansson, I.E., Bible, J., Bublitz, J., Arriola, J., Motta, C., Griswold, J., Cook, B., Sarvian, N., Clayton-Warwick, D., Wu, J., Milich, A., Oleson, M., 
Kangas, M., Pryor, M. and Krogen, P. "DE-STAR: phased-array laser technology for planetary defense and exploration," STARDUST 1st Stardust Global Virtual Workshop (SGVW-1) on Asteroids and Space Debris, Conference Proceedings edited by Massimiliano Vasile (May, 2014).

[7] Kosmo, K., Pryor, M., Lubin, P., Hughes, G.B., O’Neill, H., Meinhold, P., Suen, J., C., Riley, J., Griswold, J., Cook, B.V., Johansson, I., Zhang, Q., Walsh, K., Melis, C., Kangas, M., Bible, J., Motta, Brashears, T., Mathew, S. and Bollag, J. "DE-STARLITE - a practical planetary defense mission," Nanophotonics and Macrophotonics for Space Environments VIII, edited by Edward W. Taylor, David A. Cardimona, Proc. of SPIE Vol. 9226 (Aug, 2014).

[8] Vorontsov, M. A., Weyrauch, T., Beresnev, L. A., Carhart, G. W., Liu, L., and Aschenback, K., "Adaptive Array of Phase-Locked Fiber Collimators: Analysis and Experimental Demonstration," IEEE Journal of Selected Topics in Quantum Electronics 15, 269 (2009).

[9] Bakr, O.M. and Johnson, M. "Impact of phase and amplitude errors on array performance," Technical Report No. UCB/EECS-2009-1, http://www.eecs.berkeley.edu/Pubs/TechRpts/2009/EECS-2009-1.html (2009).

[10] Charles, C.T., "A calibrated phase and amplitude control system for phased-array transmitters," Ph.D. Dissertation, University of Washington (2006).

[11] Hansen, R.C., Phased Array Antennas, Second Edition, Wiley: New York (2009).

[12] Mailloux, R.J., Phased Array Antenna Handbook, Second Edition, Artech House: Boston (2006).

[13] Rabinovich, V., and Alexandrov, N. "Typical Array Geometries and Basic Beam Steering Methods." In Antenna Arrays and Automotive Applications (pp. 23-54). Springer: New York (2013). 